\title{
Basophil sensitivity through CD63 or CD203c is a functional measure for specific immunotherapy
}

\author{
Susan Mikkelsen ${ }^{1}$, Bo Martin Bibby², Mette Konow Bøgebjerg Dolberg ${ }^{1}$, Ronald Dahl ${ }^{1}$, Hans Jürgen Hoffmann ${ }^{1,3^{*}}$
}

\begin{abstract}
Background: Subcutaneous Immunotherapy (SCIT) modifies the allergic response and relieves allergic symptoms. SCIT is the only and a very effective treatment for insect venom allergy. We hypothesized that basophil sensitivity, measured through the basophil activation test, would decrease during SCIT up dosing. Expression of CD203C was compared to CD63 as marker for basophil activation, using a Bland Altman plot and ROC curves.

Methods: Patients $(n=18)$ starting subcutaneous SCIT for wasp allergy with an up dosing scheme of 7 to 11 weeks were enrolled. Heparinised blood samples were drawn at weeks 1-4, 7 and the first maintenance visit. Basophils were stimulated at 7 log dilutions of $V$. vespula allergen for $15 \mathrm{~min}$, and were stained with CD203c and CD63. Basophils were identified as CD203c $c^{+}$leukocytes, and the proportion of $\mathrm{CD}_{3}{ }^{+}$and $\mathrm{CD} 203 \mathrm{C}^{+}$cells were plotted against allergen concentration. A sigmoid curve was fitted to the points, and the allergen concentration at which half of the maximal activation was achieved, LC50, was calculated. In another series of experiments, LC50 calculated in whole blood (AP) was subtracted from LC50 calculated with basophils suspended in plasma from a nonatopic donor (HS) to determine the protective effect of soluble factors in blood of patients treated with SCIT.

Results: Heparin blood basophil activation was similar through CD63 and CD203c. Basophils were significantly more sensitized three weeks after initiation of SCIT compared to baseline $(p<0,01)$. The difference in LC50 increased by 1,04 LC50 units $(p=0,04)$ in patients that had just achieved maintenance dose compared with patients before initiating SCIT. When maintenance allergen concentrations had been reached, an increase in the protective plasma component was documented. Blood basophil concentration was marginally reduced by SCIT.

Conclusion: Basophil activation is a versatile and sensitive tool that measures changes in the humoral immune response to allergen during SCIT.
\end{abstract}

\section{Introduction}

Allergy to insect venom can be life threatening, and leaves patients in a continuous state of anxiety [1]. Subcutaneous immunotherapy (SCIT) is able to modify the course of allergic disease, and is the only form of protection available against this allergy [2]. The efficacy of SCIT for venom allergy has been documented in a meta analysis [3]. SCIT induces cellular increase in IL-10 [4] and changes in the humeral immune system; a transient increase in free allergen specific immunoglobulin $\operatorname{IgX}$ (mainly, but not exclusively IgG4 and IgA) and a decrease in the ratio of free allergen specific immunoglobulin to allergen specific IgE bound to effector cells (mast cells and basophil granulocytes) [4,5].

\footnotetext{
* Correspondence: hans.jurgen.hoffmann@ki.au.dk

'Department of Respiratory Diseases, Aarhus University Hospital, Aarhus,
} Denmark
The basophil activation test (BAT) is an increasingly attractive method of assessing a type I allergic response [6]. BAT has been used to measure effects of SCIT [7-9]. Although it may also have diagnostic potential, its real power emerges when it is used to determine basophil sensitivity with serial dilutions of allergen, where it correlates well with clinical symptom strength $[5,10]$. The optimal choice of activation marker has been a recurrent theme; CD63 has historical value [11], and CD203c has potential because it could be used for identification and as activation marker at the same time [12]. In addition to the blood basophils' attractiveness as surrogate marker for mast cell responses, it is a leukocyte that is recruited from blood to inflamed tissue in a type I allergic response $[13,14]$. Changes in blood basophil concentration during allergen provocation may be a useful objective marker for a type I allergic response [14]. 
We devised an objective method to measure basophil sensitivity, and hypothesised that we could use it to measure changes in basophil sensitivity and soluble Immunoglobulin induced in plasma by SCIT during the up dosing phase of SCIT for wasp venom.

\section{Methods}

\section{Patients}

The ethics committee of Aarhus County approved a project, in which patients aged 18 or older, registered to begin subcutaneous immunotherapy with Vespula Alutard (ALK-Abelló, Hørsholm, Denmark) were recruited. From January to April 2007, 20 patients were recruited and 18 patients completed the project (median age 54 years, interquartile range (IQR) 44-61 years, 8 women, 10 men). The clinical history of wasp allergy was confirmed by skin prick test (SPT) (ALK Abelló, Hørsholm, Denmark) and by measuring specific IgE with the CAP system (Phadia, Copenhagen, Denmark), with a threshold of $0,35 \mathrm{kU} / \mathrm{L}$. A SPT of $>3 \mathrm{~mm}$ was considered positive. The median concentration of specific IgE to vespula venom was $3,01 \mathrm{kU} / \mathrm{L}$, with an IQR of $1,53-7$ $\mathrm{kU} / \mathrm{L}$. Some of the patients $(\mathrm{n}=5)$ were also sensitized to bee venom with a median and range of $0,95 \mathrm{kU} / \mathrm{L}$, ranging from $0,88-1,23 \mathrm{kU} / \mathrm{L}$. One patient was negative for both wasp and bee venom in the CAP test, but had a positive prick test for wasp. In SCIT increasing doses of an allergen, the patient is sensitized to, is injected subcutaneously once a week until a maintenance dose (prescheduled 100.000 SQU) is reached. Treatment continues for 3-5 years with injections every $6^{\text {th }}-8^{\text {th }}$ weeks. Allergen injections, in the up dosing phase, can be administered as clusters (11 injections at 7 visits) [15] or after the conventional regime with one injection at each visit (16 injections at 16 visits), if there is an increased risk of adverse effects to the treatment. It is possible to shift from the cluster up dosing regimen to the conventional. Six of the 16 patients that started on our cluster regimen completed it (Table 1 ). The remaining ten patients transferred to the conventional regimen, on which four patients had started. For generation of the ROC curve, additional patients ( 3 women, 3 men) with allergy to wasp venom (specific IgE $>0,35 \mathrm{kU} / \mathrm{L}$ ), and healthy, stung controls ( 5 women, 4 men) that did not have specific IgE to wasp venom, were recruited. For determination of differences in LC50 ( $\log 10$ of the allergen concentration resulting in $50 \%$ activation of basophils) as the protective component in patient plasma (see below) at maintenance dose, additional patients receiving maintenance dose of SCIT for wasp venom allergy $(\mathrm{n}=3)$ or birch pollen allergy $(\mathrm{n}=5)$ were recruited (3 women, 5 men).

\section{Basophil activation}

For basophil activation, $4 \mathrm{ml}$ venous blood was drawn into heparin tubes before allergen injection at visits 1,2 ,

Table 1 Selected salient clinical data for each patient

\begin{tabular}{|c|c|c|c|c|}
\hline Pt nr & Treatment & Side effects & maintenance dose & Ratio CD63 activation at $0,1 / 1$ \\
\hline 1 & Traditional & fatigue & 40000 & 1,07 \\
\hline 2 & traditional & weals & 100000 & 1,05 \\
\hline 3 & traditional & weals & 100000 & 0,82 \\
\hline 4 & traditional & weals, fatigue & 100000 & 0,87 \\
\hline 5 & traditional, discontinued & heartbeat, nervousness & (20) & 0,78 \\
\hline 6 & cluster, discontinued & itchy tongue & $(1000)$ & 0,78 \\
\hline 7 & cluster & hyperventilation & 100000 & 0,65 \\
\hline 8 & cluster & none & 100000 & 0,95 \\
\hline 9 & cluster & none & 100000 & 0,66 \\
\hline 10 & cluster & weals, itch, fatigue & 100000 & 0,91 \\
\hline 11 & cluster & weals & 100000 & 0,87 \\
\hline 12 & cluster & none & 100000 & 0,71 \\
\hline 13 & cluster & weals & 60000 & 0,51 \\
\hline 14 & cluster & weals & 100000 & 1,22 \\
\hline 15 & cluster & weals, fatigue & 100000 & 1,00 \\
\hline 16 & change & weals, oedema & 100000 & 0,66 \\
\hline 17 & change & weals, fatigue & 100000 & 0,59 \\
\hline 18 & change & weals & 100000 & 2,09 \\
\hline 19 & change & weals & 100000 & 1,36 \\
\hline 20 & change & weals & 100000 & 1,13 \\
\hline
\end{tabular}

Side effects, maintenance dose and CD63 activation at $0,1 \mathrm{ug} / \mathrm{ml}$ venom $/ 1 \mathrm{ug} / \mathrm{ml}$ venom are listed against SCIT regimen (traditional 16 week up-dosing protocol/ 7 week cluster up dosing protocol/change from 7 week to 16 week protocol) applied. Where the ratio of activation exceeds 0,95 it has been shown to be predictive of side effects [7] and is printed bold. Side effects were weals around the site of injection 
$3,4,7$ and at the first visit at which maintenance dose was injected. Basophil activation was done as previously described [6]; log dilutions of allergen (freeze-dried $V$. vespula allergen, a gift from ALK-Abello, Hørsholm, DK) ranging from $10 \mathrm{ug} / \mathrm{ml}$ final concentration to 10 $\mathrm{pg} / \mathrm{ml}$ final concentration were compared with PBS only as control. Heparinised blood (100 ul) was added within 2 hours of venesection, and incubated with allergen at $37^{\circ} \mathrm{C}$ for 20 minutes. A dilution series of anti FceRI antibody CRA1 (Catalog Nr 334602, Biolegend, CA) was used as positive control (data not shown). Titrated amounts of CD63 FITC (5 ul, Invitrogen MHCD6301) and CD203c PE (5 ul, Beckmann-Coulter, IM3575) were added for the last 5 minutes. Activation was terminated by addition of $2 \mathrm{ml}$ cold Saponin solution $(0,15 \mathrm{mg} / \mathrm{ml})$ in PBS for exactly 2 minutes, followed by $0,25 \mathrm{ml}$ of $5 \%$ Methanol and 10\% Paraformaledhyde in PBS and centrifugation at $400 \mathrm{~g}$ for 8 minutes at $4^{\circ} \mathrm{C}$. Samples were washed once with PBS, suspended in PBS and 150000 events were acquired on a FACS Calibur flow cytometer within 3 hours. On each day, compensation controls were acquired for CD63 FITC and CD203c PE with comp beads (Beckton-Dickinson, San Jose, CA). Data was compensated and in the analysis, the FS:SS region containing basophils was identified by back gating on $\mathrm{CD}_{203 \mathrm{c}^{+}}$cells (figure 1 ). A threshold set at $2 \%$ positive cells in the PBS tube was used to determine percent activated cells in the tubes incubated with allergen. The fraction of positive cells is plotted against allergen concentration.

The protective effect of plasma on basophil activation was calculated as difference in LC50 in full blood (autologous plasma) and LC50 of basophils in washed blood. Washed blood was prepared by diluting $4 \mathrm{ml}$ blood with $46 \mathrm{ml}$ PBS, centrifuging and diluting again with PBS up to $50 \mathrm{ml}$. The remaining cells were suspended in heterologous plasma $(1 \mathrm{ml})$ from a nonatopic donor for 15 min. Washed blood was used in a BAT test as described above. In a control experiment, the LC50 of washed blood was reconstituted with autologous plasma was similar to that of whole blood $(n=5)$.

LC50 was calculated in R [16] by fitting a sigmoid curve to data from each visit, and determining the amount of allergen, that results in $50 \%$ of maximal activation.

\section{Generation of ROC curve}

The allergen concentration at which sensitivity and specificity were optimal for CD63 and for CD203c was determined with data from 24 clinically diagnosed wasp allergic patients and 9 stung controls with no specific IgE to vespula allergen, using plots of sensitivity and

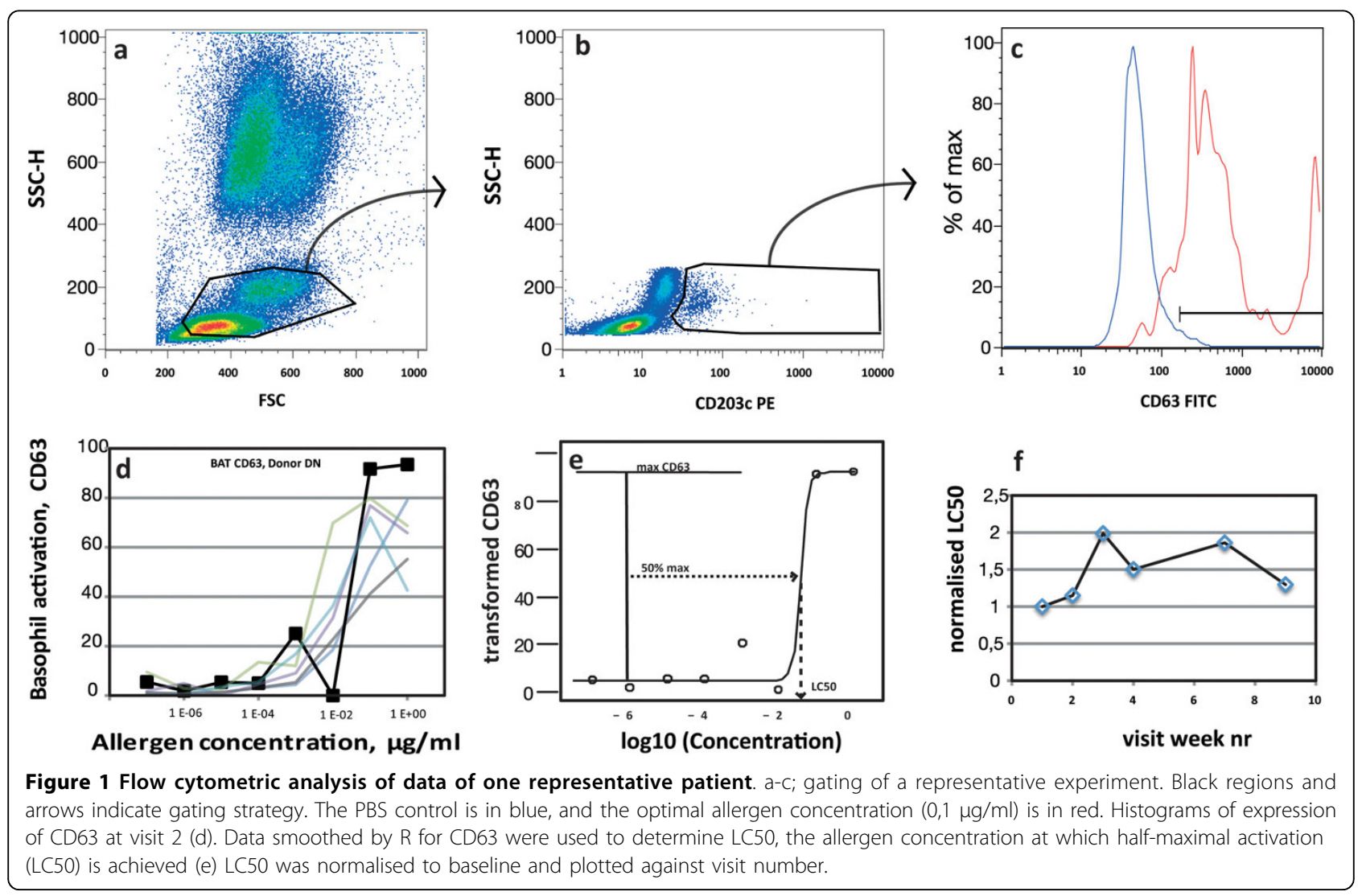


specificity [17], and confirmed with web based ROC software [18]. For SCIT patients, baseline visits were used for this analysis.

\section{Bland Altman Analysis}

The difference in basophil activation as measured by upregulation of $\mathrm{CD} 63$ and $\mathrm{CD} 203 \mathrm{c}$ was investigated by a Bland Altman analysis [19].

\section{Blood basophil concentrations determined by constant volume acquisition (CVA)}

Blood basophil concentration was determined by CVA [20]. Data is acquired for a constant, calibrated time, which appears to give a more precise measurement of volume than measurement with micro beads [20]. Briefly, 50 ul of blood was labelled with Lin1 FITC (BD Catalog Nr 340546), CD123 PE (BD Catalog Nr 340545), CD45 PE Cy7 (BD Catalog Nr 555484) and HLA DR APC (BD Catalog Nr 559866) for 15 min at room temp, and lysed with FACS Lyse (BD Catalog $\mathrm{Nr}$ 349202). Data was acquired for 4 minutes from all patients at all visits. Each day data was acquired, compensation controls were acquired, and data was compensated before analysis. Leukocytes were identified on a CD45 vs. SS plot. Basophils were identified as CD45 dim Lin $1^{\text {neg }}$ CD $123^{\text {bright }}$ HLA DR ${ }^{\text {neg }}$ cells, and the absolute concentration was calculated.

\section{Results}

Increase in basophil sensitivity during the up dosing phase of SCIT may be limited by factors in plasma The effect of the up-dosing phase of subcutaneous immunotherapy on basophil up regulation of CD63 and CD203c (figure 1) was evaluated by fitting a sigmoid curve to data points obtained with a 7-step logarithmic dilution series of $V$. vespula allergen and blood of wasp allergic patients drawn at each of visits 1, 2, 3, 4, 7 and maintenance visit, if that did not coincide with visit 7 . From this curve at each visit, the concentration of allergen resulting in half-maximal CD63 activation was determined (figure 1e). It is termed LC50. When normalised to the baseline visit, the LC50 was normally distributed. When plotted against visit, it increased significantly from the first to the third week of immunotherapy (visit $1-3 ; \mathrm{p}=0,0098$, visit $2-3 \mathrm{p}=0$, 0259 , visit $3-4 \mathrm{p}=0$, 0274, visit 3 -maint $\mathrm{p}=0,0095$ for all in a mixed linear model, $\mathrm{p}=0,029$ for CD63, $\mathrm{p}=$ 0,015 for CD203c, $\mathrm{n}=18$ ), and tended toward baseline levels beyond that (figure 2a).

In a cross-sectional study to determine the contribution of humoral factors toward BAT performed in whole blood, blood from different patients initiating SCIT and patients on maintenance was washed with PBS and replaced by heterologous serum (termed HS in contrast to autologous plasma, AP) from a nonatopic donor as described in methods, and was used in a BAT. Replacing AP with HS dramatically increased basophil sensitivity. At baseline the sensitivity increased by 2,576 LC50 units ( $\mathrm{n}=8, \mathrm{p}<0,001)$, and at maintenance it increased by 3,595 LC50 units $(\mathrm{n}=10, \mathrm{p}<0,001)$ (figure 2b). At the early stage of maintenance, the reduction in basophil sensitivity resulting from the autologous plasma had thus increased by 1,02 LC50 units ( $\mathrm{p}=0,04)$ (figure 2c).

\section{CD63 and CD203c are equivalent markers for basophil activation}

The optimal allergen concentration used to detect allergy to $V$. vespula allergen was determined with sensitivity vs. specificity curves and is illustrated with a ROC curve (figure 3a) with 24 patients with documented allergy and 9 exposed controls. The optimal final $V$. vespula allergen concentration was $0,1 \mu \mathrm{g} / \mathrm{ml}$. The sensitivity and specificity were $88 \%$ and $78 \%$ for CD63 at 3,28\% basophil activation. For CD203c, the sensitivity and specificity were $88 \%$ and $89 \%$ at $3,85 \%$ basophil activation.

Agreement between activation measurements performed with CD63 as the predicate method and CD203c determined with a Bland Altman plot [19] (figure 3b). The median difference of CD63-CD203c was $-1,2 \%$, IQR $23,5 \%$ to $-28,6 \%$ (not normally distributed, $\mathrm{n}=$ 914).

Difference between CD63 and CD203c was determined with an approximate $\mathrm{T}$ test in a linear mixed model. For all concentrations there was no significant difference between CD63 and CD203c with all p-values greater than 0,11 . When autologous plasma is replaced with heterologous serum, the value of CD63-CD203c increased from $4,2 \%$ (IQR -20, $3-31,7$ ) to $41,7 \%$ (IQR $8,3-73,5, \mathrm{n}=135$. In the same linear mixed model that takes account for repeated measures the relative difference between activation through CD63 and CD203c was larger after plasma was replaced with serum $(\mathrm{p}=0,0172)$. It increased significantly $(\mathrm{n}=15, \mathrm{p}$ $<0,05)$ at four of the six highest allergen concentrations, suggesting that CD203c is up regulated less than CD63 when autologous plasma is replaced by heterologous serum. This was not due to an increase in baseline MFI for CD203c, as the baseline MFI for CD63 and CD203c increased 1,92 and 1,83 fold, respectively, after replacement of plasma with serum. This was not significant.

\section{The blood basophil concentration decreases marginally during SCIT}

Blood basophil concentration measured using CVA was 37 (IQR $28-55, \mathrm{n}=18$ ) cells/ $\mu \mathrm{l}$ in allergic patients treated with SIT, and 35 (IQR $23-42, \mathrm{n}=6, \mathrm{p}=0,06$ ) cells $/ \mu \mathrm{l}$ in controls. It increased by median 1 (IQR -2 - 

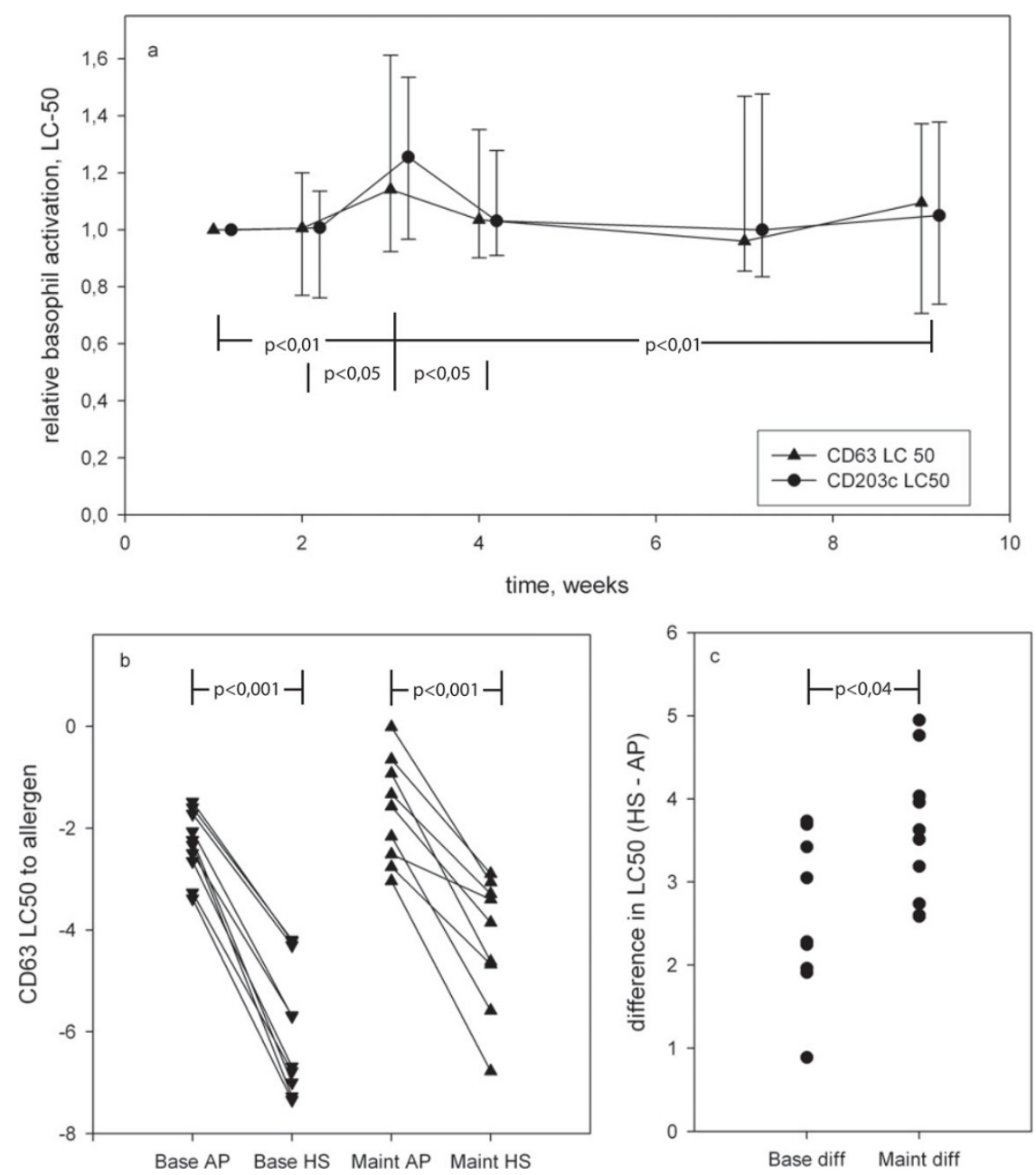

Figure 2 The effect of SCIT on basophil activity. (a) Median LC50 is plotted against visit for patients on SCIT ( $n=18$ ). At visit 3 basophils are significantly more sensitive than at other visits. (b) The protective effect of plasma is illustrated by replacing plasma with serum from a nonallergic donor. (c) The net protective effect measured as change in LC50 between basophils activated in autologous serum and in heterologous plasma is larger in patients on maintenance dose $(n=9)$ than in patients initiating SCIT $(n=10)$.

6) cell $/ \mu \mathrm{l}(5 \%)$ per 30 minutes for patients receiving allergen injections and by median 3 (IQR 2 - 5) cells/ $\mu$ l (12\%) per 30 minutes in control persons after correction for time between sampling. The difference was significant in patients and controls when measured over 90 and 120 minutes $(\mathrm{p}<0,04)$, but not when measured over 30 minutes.

Discordance between anamnesis, slgE, prick test and BAT On the scale proposed by Brown [21], 14 patients reported moderate symptoms and 4 patients reported severe symptoms when stung. Seven patients had other allergies (2 latex, 3 bee, 2 cat, 3 house dust mites, 1 perfume, 1 kiwi, 1 nuts, 1 apples) and other allergic disease (3 asthma, 3 hay fever, 3 Urticaria).

For two patients the activity of the basophils for both CD203c and CD63 was only just above the threshold values (i.e. positive) at some of the visits. These two patients had almost no symptoms/adverse effects to treatment with SIT, but they had moderate symptoms as response to wasp sting, specific $\operatorname{IgE}$ at 3,01 $\mathrm{kU} / \mathrm{L}$ and $6,0 \mathrm{kU} / \mathrm{L}$ respectively and positive skin prick tests (SPT) before the beginning of SIT. One patient had moderate symptoms to wasp sting and positive skin prick test but negative specific IgE against wasp before the beginning of SIT. The BAT of this participant was positive at all visits, and the patient had local reactions to the treatment and a systemic reaction at visit 2 .

\section{Discussion}

We have monitored changes in basophil sensitivity as the logarithm of the concentration of allergen that elicits a half-maximal response, and blood basophil concentration, during the up dosing phase of SIT. We have found 


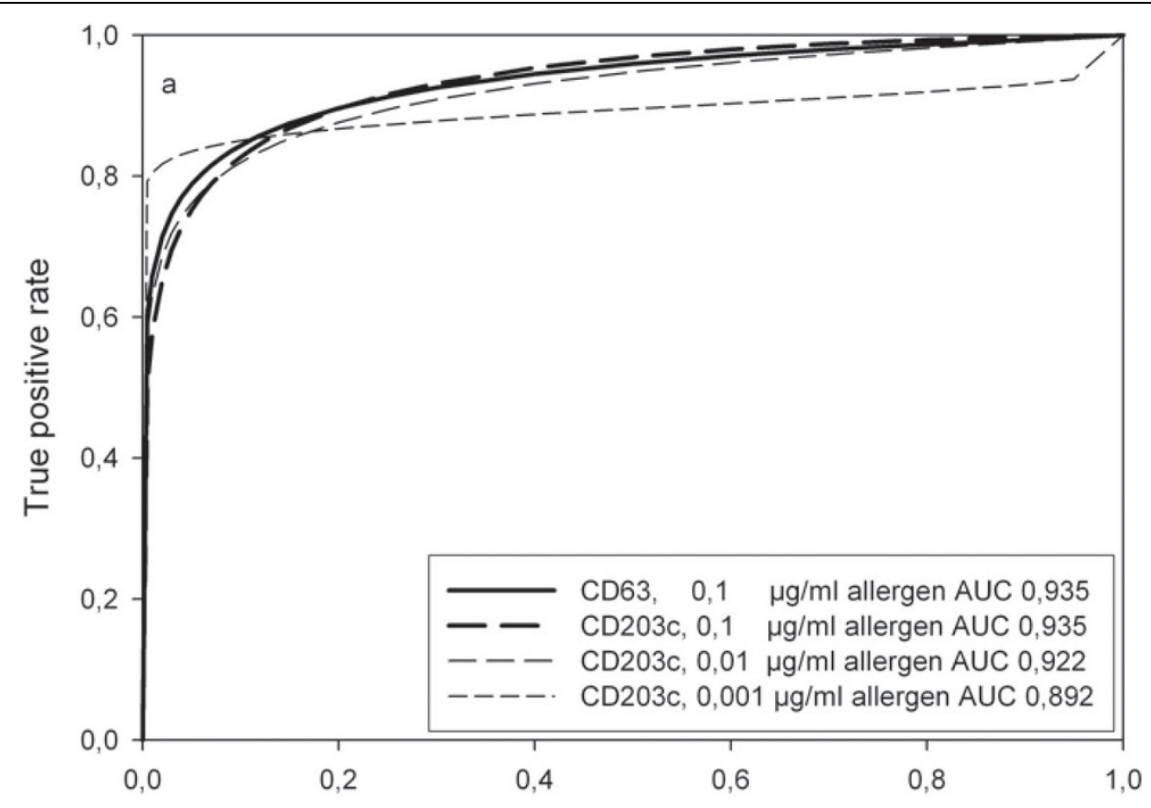

False positive rate

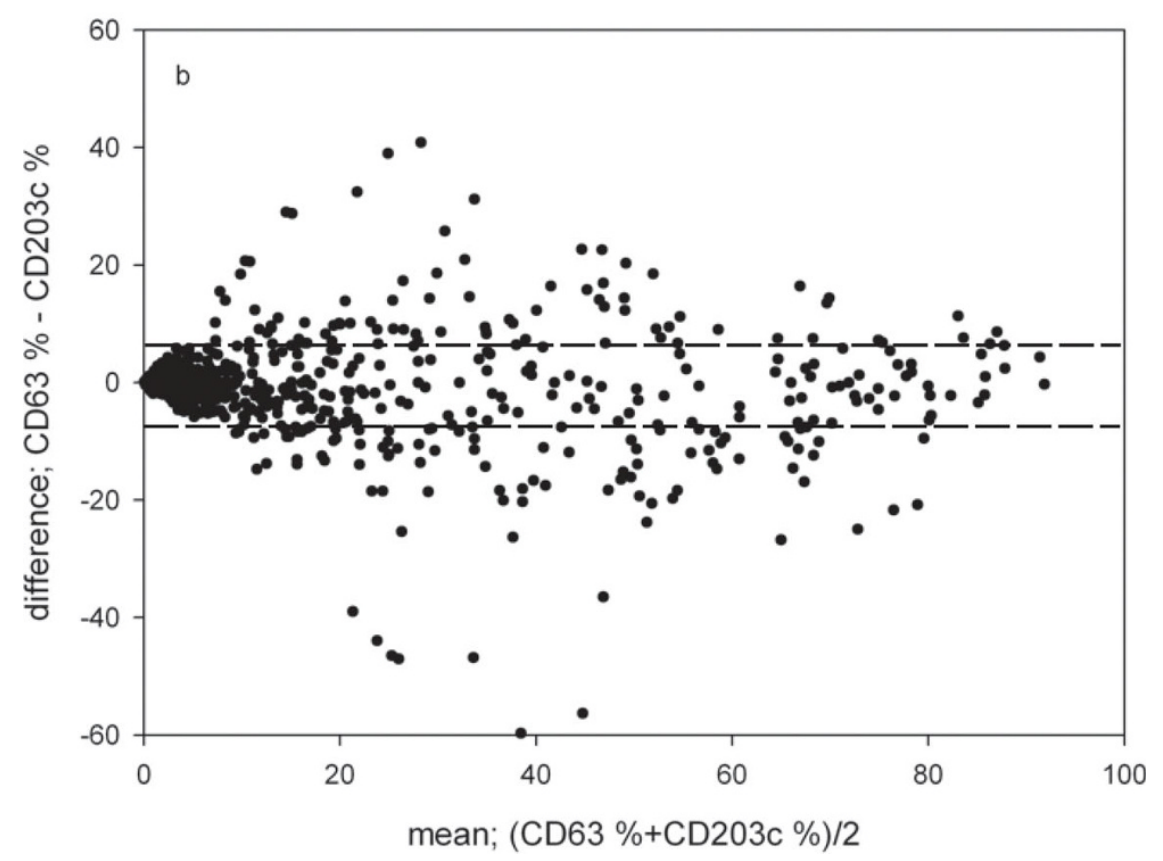

Figure 3 Comparison of CD63 and CD203c as readout. (a) the ROCs for CD63 and CD203C at the optimal concentration of allergen, 0,1 $\mu \mathrm{g} /$ $\mathrm{ml}$, were indistinguishable. (b) Bland Altman plot of CD63-CD203c. Stippled lines indicate $25^{\text {th }}$ and $75^{\text {th }}$ quartiles.

that there is a transient increase in basophil sensitivity after 3 weeks of SIT. This increase in sensitivity may reflect competing amounts of immunoglobulin on basophils (IgE) and free immunoglobulin in circulation (IgX, all classes of immunoglobulin with affinity for haptens on allergen). It coincides with the early decrease in basophil histamine release and increase in free IgG4 and IgA described recently [4].
During ultra rush procedures of SCIT with Japanese cedar [9] or birch or cat [8], maximal expression of CD203c as \% activated cells on basophils [9] or MFI [8] after cross linking with allergen decreased with time. BAT could not predict results of a sting challenge at 0,1 or 1,0 ug venom [22]. In a 5-day rush up dosing regimen, BAT at suboptimal concentrations appeared elevated after 5 days corresponding to the findings after 3 
weeks in the present 16-week regimen (the significance of this had not been tested). Basophil activation was significantly reduced after six months and after three years [23]. SCIT for birch or grass allergy significantly decreased CD-sens (analogous to LC50) tenfold [5], where the present results did not show a significant change. This may be because in the present experiment, BAT was done during the first 7 weeks assuming that all patients would complete up dosing by a semi rush protocol. Only 10 of 20 patients completed the semirush protocol. The protective component in plasma (that corresponds to Allergen Binding Activity (ABA)) increased by 1,04 LC50 units. This corresponds well to the tenfold change in ABA published recently [5].

Change in basophil activation measured as CD63 or CD203c activation at $0,1 \mathrm{ug} / \mathrm{ml}$ allergen did not vary significantly during the up dosing phase. A ratio of CD63 activation at $0,1 \mathrm{ug} / \mathrm{ml} / 1 \mathrm{ug} / \mathrm{ml}>92 \%$ has previously been shown to associate with severe side effects [7]. In this study, 8 patients had a ratio $>92 \%$. This did not associate with side effects (Table 1). In a study of modified rush up dosing of insect venom, the ratio of basophil activation at $0,1 \mathrm{ug} / \mathrm{ml}$ venom $/ 1 \mathrm{ug} / \mathrm{ml}$ venom above $92 \%$ at baseline predicted severe side effects during up dosing [7]. In the present study, 8 of 18 patients had a ratio of basophil activation predicting severe side effects. Neither others [24] nor we could confirm the usefulness of this ratio.

The up dosing scheme in the present study is much slower, and associated with very few side effects. This may explain that we do not find the decrease that was observed in the other studies, and why there were no side effects to associate with a high ratio of activation. The ratio of cell bound IgE to total free immunoglobulin may be the most significant effect parameter, which determines whether a basophil activation test becomes positive. The protective effect of SCIT in patient plasma (which we assume contains immunoglobulins competing for epitopes on allergen) had increased by $>1$ LC50 unit when comparing patients at baseline with patients just initiating maitenance therapy. Basophil activation has been shown to correlate well with clinical measures [10]. Our study did not extend long enough to demonstrate an effect of treatment on basophil activation by flow cytometry, which has been shown by others [5]. Inhibition of histamine release, the gold standard for basophil activity, was significantly different from baseline after 6 weeks of SCIT [4]. Whilst single concentration analyses of basophil activation may not be sufficient to monitor effects of SCIT [22], we confirm here that this can be done with logarithmic series of dilutions of allergen.

CD-sens is the fist algorithm developed to measure basophil sensitivity in a basophil activation test [25].
The allergen dose giving maximal activation is determined, and a line of regression is calculated through that point and the activation rates at the two preceding allergen concentrations. The half-maximal activation is determined from this interpolated line. CD-sens [25] was evaluated as a technique to calculate basophil sensitivity, but was not found to be useful in our hands because the allergen concentrations we used were too low to obtain a maximal activation as most of the curves we obtained were not bell shaped, and because our log dilutions were too widely spaced to obtain accurate CDsens. LC50 considers more values than CD-sens, and may be less subjective. The values obtained with LC50 were larger than those obtained with CD-sens.

The present data gave us an opportunity to determine the optimal allergen concentration for diagnosing wasp allergy with a ROC curve to be $0,1 \mathrm{ug} / \mathrm{ml}$ final concentration in the reaction vessel with blood, which compares well with $0,5 \mathrm{ug} / \mathrm{ml}$ [22] and 0,5 - 0,05 ug/ml [23] final concentration of $\mathrm{V}$ vespula allergen in BAT published by others. The ROC curves for CD63 and CD203c and the threshold for positivity were almost identical for CD63 and CD203c, suggesting that the two markers measure similar aspects of basophil activation. Although Boumiza has disputed this [26], it has since been confirmed by a number of clinically oriented publications $[24,27,28]$. We have previously shown that choice of fluorochome and lysis procedure had favoured CD203c over CD63, illustrating the need to consider all aspects of an experiment [6]. We have done the first Bland Altman analysis of CD63 and CD203c to show that in full blood there is no difference between the markers, whereas there is significantly less up regulation of CD203c than of CD63 on washed basophils. The effect appears not to be due to increased baseline expression of CD203c, as this was similar for both markers. Washing away plasma immunoglobulins increases the sensitivity of the test significantly, but masks the clinical relevance as the total response is compounded from the free immunoglobulin absorption of allergen and the cross linking of cell bound IgE.

The basophil numbers we obtained using CVA were within a recently published reference interval [29]. Previously, we had shown that ingestion of allergen resulted in a $20 \%$ reduction of blood basophil concentration compared with placebo [14]. Allergen injection, as a negative control, resulted in a marginal reduction in blood basophil numbers compared with controls. Changes in blood basophil concentration may thus be a marker for type I allergic response. As the difference in blood concentration of basophils increased with time from $30 \mathrm{~min}$ to $120 \mathrm{~min}$, the optimal time frame for measuring blood basophil concentration as marker for a type I allergic reaction remains to be determined. 
Discordance between in vivo, ex vivo and in vitro tests is a recurrent problem in allergen diagnosis, and some have suggested that basophil activation should be the arbitrating test if anamnesis and specific IgE do not support the same conclusion [30]. We found a rate of discordance between patient history and in vitro diagnostic tests ( 3 patients of 18 with discordance between patient history and in vitro diagnostic tests) that was similar to others $[23,30]$. The fact that basophil activation in full blood correlates with clinical symptoms and does reflect the response by cell-bound IgE in the context of competing immunoglobulins argues that it may occupy a special niche amongst diagnostic tests for allergy.

\section{Conclusion}

Basophil sensitivity through CD63 or Cd203c increases comparably during the first weeks of specific immunotherapy, but can measure a protective element already when maintenance dose is reached.

\section{Acknowledgements}

We would like to thank the participating patients and nurses and technician Anne Marie Toft for excellent technical assistance. Financial support by the Velux Foundation and Aarhus University is gratefully acknowledged.

\section{Author details}

'Department of Respiratory Diseases, Aarhus University Hospital, Aarhus, Denmark. ${ }^{2}$ Department of Biostatistics, Faculty of Medicine, University of Aarhus, Aarhus, Denmark. ${ }^{3}$ Clinical Institute, Faculty of Medicine, University of Aarhus, Aarhus, Denmark.

\section{Authors' contributions}

SM did most experiments, analysed the data and wrote the first draft of the manuscript. MKBD did some experiments. BMB designed and performed statistical analyses. RD contributed to the study design and participated in analysis and writing. HJH conceived the study and wrote the manuscript. All authors have read and approved the final manuscript.

\section{Competing interests}

The authors declare that they have no competing interests.

Received: 19 December 2009

Accepted: 16 February 2010 Published: 16 February 2010

\section{References}

1. Oude Elberink JN, De Monchy JG, Heide Van Der S, Guyatt GH, Dubois AE: Venom immunotherapy improves health-related quality of life in patients allergic to yellow jacket venom. J Allergy Clin Immunol 2002, 110:174-182

2. Canonica GW, Baena-Cagnani CE, Bousquet J, Bousquet PJ, Lockey RF, Malling HJ, Passalacqua G, Potter P, Valovirta E: Recommendations for standardization of clinical trials with Allergen Specific Immunotherapy for respiratory allergy. A statement of a World Allergy Organization (WAO) taskforce. Allergy 2007, 62:317-324.

3. Ross RN, Nelson HS, Finegold I: Effectiveness of specific immunotherapy in the treatment of hymenoptera venom hypersensitivity: a metaanalysis. Clin Ther 2000, 22:351-358.

4. Francis JN, James LK, Paraskevopoulos G, Wong C, Calderon MA Durham SR, Till SJ: Grass pollen immunotherapy: IL-10 induction and suppression of late responses precedes lgG4 inhibitory antibody activity. J Allergy Clin Immunol 2008, 121:1120-1125.

5. Nopp A, Cardell LO, Johansson SG, Oman H: CD-sens: a biological measure of immunological changes stimulated by ASIT. Allergy 2009, 64:811-814.
6. Hoffmann HJ, Bogebjerg M, Nielsen LP, Dahl R: Lysis with Saponin improves detection of the response through CD203C and CD63 in the basophil activation test after crosslinking of the high affinity lgE receptor FcepsilonRI. Clin Mol Allergy 2005, 3:10.

7. Kosnik M, Silar M, Bajrovic N, Music E, Korosec P: High sensitivity of basophils predicts side-effects in venom immunotherapy. Allergy 2005, 60:1401-1406.

8. Plewako H, Wosinska K, Arvidsson M, Bjorkander J, Skov PS, Hakansson L, Rak S: Basophil interleukin 4 and interleukin 13 production is suppressed during the early phase of rush immunotherapy. Int Arch Allergy Immunol 2006, 141:346-353.

9. Nagao M, Hiraguchi Y, Hosoki K, Tokuda R, Usui T, Masuda S, Yamaguchi M, Fujisawa T: Allergen-induced basophil CD203c expression as a biomarker for rush immunotherapy in patients with Japanese cedar pollinosis. Int Arch Allergy Immunol 2008, 146(Suppl 1):47-53.

10. Nopp A, Johansson SG, Ankerst J, Bylin G, Cardell LO, Gronneberg R, Irander K, Palmqvist M, Oman H: Basophil allergen threshold sensitivity: a useful approach to anti-IgE treatment efficacy evaluation. Allergy 2006, 61:298-302.

11. Knol EF, Mul FP, Jansen H, Calafat J, Roos D: Monitoring human basophil activation via CD63 monoclonal antibody 435. J Allergy Clin Immunol 1991, 88:328-338.

12. Buhring HJ, Seiffert M, Giesert C, Marxer A, Kanz L, Valent P, Sano K: The basophil activation marker defined by antibody $97 \mathrm{~A} 6$ is identical to the ectonucleotide pyrophosphatase/phosphodiesterase 3. Blood 2001, 97:3303-3305.

13. Nouri-Aria KT, Irani AM, Jacobson MR, O'Brien F, Varga EM, Till SJ, Durham SR, Schwartz LB: Basophil recruitment and IL-4 production during human allergen-induced late asthma. J Allergy Clin Immunol 2001, 108:205-211

14. Hoffmann HJ, Skjold T, Raithel M, Adolf K, Hilberg O, Dahl R: Response of respiratory flour allergics in an ingested flour challenge may involve plasmacytoid dendritic cells, CD25+ and CD152+ T cells. Int Arch Allergy Immunol 2006, 140:252-260.

15. Blumberga G, Groes L, Haugaard L, Dahl R: Steroid-sparing effect of subcutaneous SQ-standardised specific immunotherapy in moderate and severe house dust mite allergic asthmatics. Allergy 2006, 61:843-848.

16. R: A Language and Environment for Statistical Computing. http://www.Rproject.org.

17. Greiner M: Two-graph receiver operating characteristic (TG-ROC): a Microsoft-EXCEL template for the selection of cut-off values in diagnostic tests. J Immunol Methods 1995, 185:145-146.

18. ROC analysis: web-based calculator for ROC curves. Baltimore. http:// www.jrocfit.org.

19. Glencross D, Scott LE, Jani IV, Barnett D, Janossy G: CD45-assisted PanLeucogating for accurate, cost-effective dual-platform CD4+ T-cell enumeration. Cytometry 2002, 50:69-77.

20. Hansen S, Dahl R, Hoffmann HJ: Determination of blood leukocyte concentration with constant volume acquisition on a flow cytometer is comparable to individualized single platform testing with beads as internal reference standard. J Immunol Methods 2008, 338:58-62.

21. Brown SG: Clinical features and severity grading of anaphylaxis. J Allergy Clin Immunol 2004, 114:371-376.

22. Erdmann SM, Sachs B, Kwiecien R, Moll-Slodowy S, Sauer I, Merk HF: The basophil activation test in wasp venom allergy: sensitivity, specificity and monitoring specific immunotherapy. Allergy 2004, 59:1102-1109.

23. Ebo DG, Hagendorens MM, Schuerwegh AJ, Beirens LM, Bridts CH, De Clerck LS, Stevens WJ: Flow-assisted quantification of in vitro activated basophils in the diagnosis of wasp venom allergy and follow-up of wasp venom immunotherapy. Cytometry B Clin Cytom 2007, 72:196-203.

24. Eberlein-Konig B, Varga R, Mempel M, Darsow U, Behrendt H, Ring J: Comparison of basophil activation tests using CD63 or CD203C expression in patients with insect venom allergy. Allergy 2006, 61:1084-1085.

25. Johansson SG, Nopp A, van Hage M, Olofsson N, Lundahl J, Wehlin L, Soderstrom L, Stiller V, Oman H: Passive IgE-sensitization by blood transfusion. Allergy 2005, 60:1192-1199.

26. Boumiza R, Monneret G, Forissier MF, Savoye J, Gutowski MC, Powell WS, Bienvenu J: Marked improvement of the basophil activation test by detecting CD203c instead of CD63. Clin Exp Allergy 2003, 33:259-265. 
27. Ocmant A, Peignois $Y$, Mulier S, Hanssens L, Michils A, Schandene L: Flow cytometry for basophil activation markers: the measurement of CD203C up-regulation is as reliable as CD63 expression in the diagnosis of cat allergy. J Immunol Methods 2007, 320:40-48.

28. Abuaf N, Rostane H, Rajoely B, Gaouar H, Autegarden JE, Leynadier F, Girot R: Comparison of two basophil activation markers CD63 and CD203C in the diagnosis of amoxicillin allergy. Clin Exp Allergy 2008, 38:921-928.

29. Ducrest S, Meier F, Tschopp C, Pavlovic R, Dahinden CA: Flowcytometric analysis of basophil counts in human blood and inaccuracy of hematology analyzers. Allergy 2005, 60:1446-1450.

30. Ebo DG, Hagendorens MM, Bridts CH, De Clerck LS, Stevens WJ: Hymenoptera venom allergy: taking the sting out of difficult cases. $J$ Investig Allergol Clin Immunol 2007, 17:357-360.

doi:10.1186/1476-7961-8-2

Cite this article as: Mikkelsen et al: Basophil sensitivity through CD63 or CD203C is a functional measure for specific immunotherapy. Clinical and Molecular Allergy 2010 8:2.

\section{Submit your next manuscript to BioMed Central} and take full advantage of:

- Convenient online submission

- Thorough peer review

- No space constraints or color figure charges

- Immediate publication on acceptance

- Inclusion in PubMed, CAS, Scopus and Google Scholar

- Research which is freely available for redistribution

Submit your manuscript at www.biomedcentral.com/submit 